\title{
Influence of the probiotic Levusell SB Plus on the productive qualities of hens in broiler breeders
}

\author{
Elena Pilyukshina ${ }^{1 *}$, Vladimir Khaustov $^{1}$, Valentina Rusanova ${ }^{1}$, Alexey Popelyaev ${ }^{1}$, \\ Elena Mashkina ${ }^{1}$, and Vladislav Sarychev ${ }^{1}$ \\ ${ }^{1}$ Altai State Agricultural University, 98, Krasnoarmejskij ave., Barnaul, 656049, Russia
}

\begin{abstract}
More and more probiotics are used in poultry feeding. Understanding that the inclusion of beneficial microorganisms in the diet contributes to the normalization of intestinal microflora serves to prevent metabolic disorders, improves the activity of the gastrointestinal tract, increases immunity and, as a result, has a positive effect not only on the health of the bird, but also on its productivity indicators. Therefore, the purpose of our study was to establish the effect of the probiotic Levusell SB Plus on the productive qualities of hens in broiler breeders. The experiment was carried out in the production conditions of Kamenskaya Poultry Farm LLC, Kamensky District, Altai Territory on hens of the parent stock of the Ross 308 cross. The use of the probiotic Levusell SB Plus at a dose of $1.0 \mathrm{~kg} / \mathrm{t}$ as a part of the complete feed for laying hens of the parent stock at the age of 23 to 63 weeks led to an increase in poultry livability, an increase in the total egg production and the intensity of egglaying capacity. The addition of a probiotic to the poultry ration contributed to the optimization of the egg weight, an increase in the yield of hatching eggs, an increase in the hatch of chickens and the yield of broilers per initial layer by $3.9 \%$.
\end{abstract}

\section{Introduction}

Poultry farming is a highly developed branch of animal husbandry, dynamically developing and providing the population with valuable dietary food products - eggs and meat.

The share of poultry meat in Russia is the leading one among all types of meat. In this regard, an increase in poultry production, as a rule, requires an improvement in the quality of poultry feeding, including through the use of probiotics $[1,2]$.

Probiotics have a positive effect on the bird's body. Moreover, they are components of a healthy diet, serve to normalize the intestinal microflora, prevent metabolic disorders, improve the activity of the gastrointestinal tract and increase immunity $[3,4,5,6]$. Numerous studies have revealed a positive effect of various probiotics on the productive qualities of broiler chickens $[7,8,9,10]$ and laying hens $[11,12]$. Only a few studies are

\footnotetext{
*Corresponding author: lexx-74@bk.ru
} 
devoted to the use of probiotics in feeding rearing young birds and parent flock of table poultry $[13,14]$.

Therefore, revealing the effectiveness of the use of certain probiotics in the diets of broiler breeders is an urgent and poorly studied area.

The purpose of this study is to establish the effect of the probiotic Levusell SB Plus on the productivity of hens in broiler breeders.

\section{Materials and Methods}

The experiment was carried out in the conditions of Kamenskaya Poultry Farm LLC, Kamensky District, Altai Territory on hens of the parent stock of the Ross 308 cross-breed. In this case, two experimental groups of 8331 heads were formed. The first group was the control group and received a basic diet corresponding to the recommendations of the cross. The second experimental group, in addition to the main diet, received the probiotic Levusell SB Plus at a dose of $1.0 \mathrm{~kg} / \mathrm{t}$.

This probiotic is a pure dry active yeast of the Saccharomyces cerevisiae type boulardii, microencapsulated and covered with a protective shell. Yeast of this strain displace pathogenic microflora and stimulate the growth of beneficial microorganisms in the gastrointestinal tract, help strengthening the intestinal wall, optimize the immune system, and ultimately increase the livability and productivity of poultry, which has been confirmed by studies on broiler chickens $[15,16,17]$.

The duration of the experiment was from 23 to 63 weeks of age. The poultry was kept on the floor, and the zoohygienic conditions corresponded to modern requirements.

The obtained data were subjected to statistical processing using a personal computer and Microsoft Office Excel package.

\section{Results}

The main purpose of the parent flock is to obtain the hatching egg, its quantity is determined by the number of laying hens. Livability is determined based on the number of culled and dead birds (Table 1).

Table 1. Livability of breeder hens.

\begin{tabular}{|l|c|c|}
\hline \multirow{2}{*}{ Indicator } & \multicolumn{2}{c|}{ Group } \\
\cline { 2 - 3 } & control & experimental \\
\hline Initial livestock, heads & 8331 & 8331 \\
\hline Died, goal. & 457 & 279 \\
\hline Culled, goal & 268 & 278 \\
\hline Total eliminated birds, heads & 725 & 557 \\
\hline Final livestock, heads & 7606 & 7774 \\
\hline Livability,\% & 91.2 & 93.3 \\
\hline Difference with control group,\% & - & 2.1 \\
\hline
\end{tabular}

Analysis of Table 1 showed that the initial livestock in the experimental groups was the same - 8331 heads in each group. During the period of the experiment, 725 heads were eliminated in the control group, which is 168 heads or $23 \%$ more than in the experimental group. At the same time, approximately the same number of birds, 268-278 heads, were culled in both groups. The number of dead birds in the second group fed with the probiotic was 1.6 times less than in the control one, which can be explained by the positive effect of this drug. 
Thus, the greatest livability of chickens was observed in the experimental group and amounted to $93.3 \%$, which is $2.1 \%$ more than in the control group.

The influence of the probiotic Levusell SB Plus on the total egg production is presented in Table 2.

The results of Table 2 indicate that the peak of the total egg production falls on the second month of laying. At the same time, at the beginning of the experiment, less eggs were collected from the experimental group by $5.9-0.3 \%$, than in the control group. From the third month of laying, the situation changed and subsequently the superiority of the experimental group over the control group was $0.8-9.0 \%$.

In total, over the entire period of laying, 1,600,350 eggs were produced by the hens of the control group, while 42,535 eggs or $2.7 \%$ more were produced by the hens of the experimental group.

Table 2. Total egg production from laying hens of the parent stock, pcs.

\begin{tabular}{|c|c|c|}
\hline \multirow{2}{*}{ Bird age, weeks } & \multicolumn{2}{|c|}{ Group } \\
\cline { 2 - 3 } & control & experimental \\
\hline $23-27$ & 129090 & 121530 \\
\hline $28-32$ & 258240 & 257400 \\
\hline $33-37$ & 246390 & 248370 \\
\hline $38-42$ & 232440 & 233310 \\
\hline $43-47$ & 212910 & 218730 \\
\hline $48-52$ & 192990 & 204780 \\
\hline $53-57$ & 169020 & 185160 \\
\hline $58-63$ & 159270 & 173605 \\
\hline \multicolumn{1}{|c|}{} & 1600350 & 1642885 \\
\hline $\begin{array}{l}\text { Dotal } \\
\text { controrence with }\end{array}$ & - & 42535 \\
\hline $\begin{array}{l}\text { In \% to control } \\
\text { group }\end{array}$ & - & 102.7 \\
\hline
\end{tabular}

Based on the data on the total egg production and the number of laying hens, the intensity of egg-laying capacity is calculated. Changes in the intensity of egg-laying capacity throughout the experiment are shown in Fig. 1.

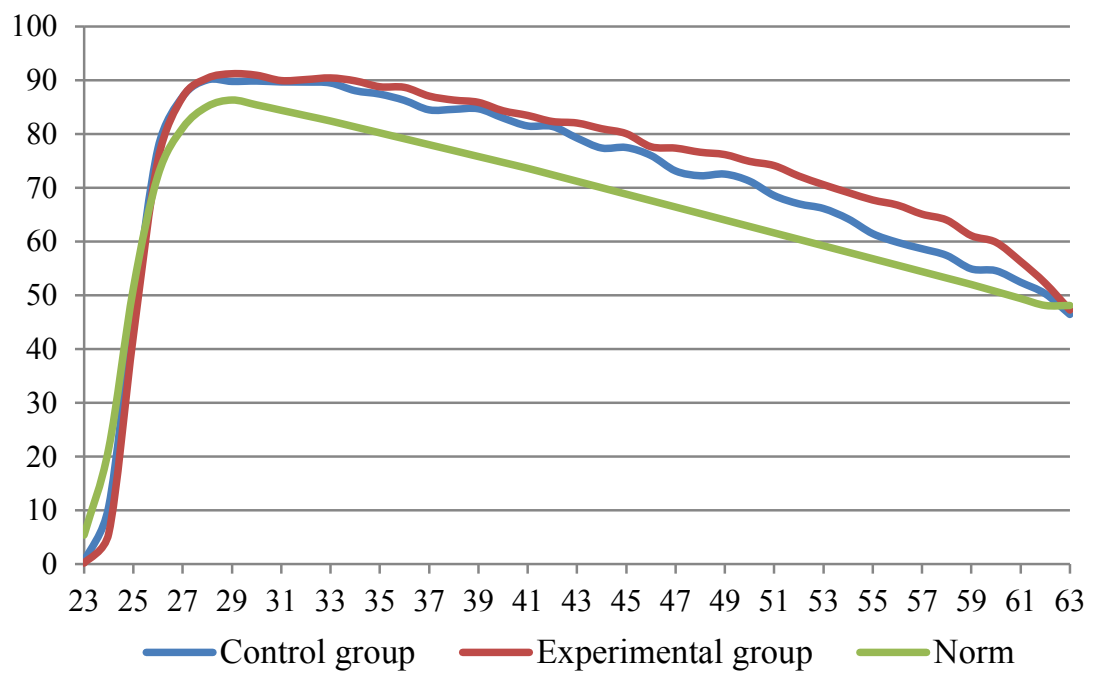

Fig. 1. Intensity of egg-laying capacity, $\%$. 
Fig. 1 shows that the intensity of egg-laying capacity in the experimental groups up to 25 weeks of age was $4.8-15.8 \%$ lower than the norm. Later, in the control group, this indicator exceeded the norm by $2.2-9.0 \%$, and in the experimental group - by $2.9-12.2 \%$.

The control group had higher indicators of the intensity of egg-laying capacity compared to the experimental group at the beginning of the experiment from 23 to 27 weeks of age. From 28 to 63 weeks of age, layers of the experimental group outperformed their peers from the control group by $0.3-6.9 \%$. The most significant difference between the experimental and control groups was observed at the end of the experiment from 47 to 60 weeks of age (4.2-6.9\%). On average, the intensity of egg-laying capacity in the experimental group was $72.7 \%$, which is $2.4 \%$ more than in the control group and $7.5 \%$ more than the norm.

An important indicator characterizing the suitability of eggs for hatching is their weight. Both too small and too large eggs are not left for hatching. The weight of a day-old chick directly depends on the size of the eggs. Table 3 shows the data on the effect of the probiotic on the weight of eggs obtained from laying hens of a broiler breeder flock.

Table 3. Egg weight, g.

\begin{tabular}{|c|c|c|c|}
\hline Bird age, weeks & \multirow{2}{*}{ Norm } & \multicolumn{2}{|c|}{ Group } \\
\cline { 3 - 4 } & & control & experimental \\
\hline 23 & 49.0 & $47.5 \pm 0.81$ & $47.0 \pm 0.73$ \\
\hline 27 & 55.0 & $55.8 \pm 0.77$ & $54.6 \pm 0.91$ \\
\hline 32 & 59.8 & $59.7 \pm 0.98$ & $59.2 \pm 0.88$ \\
\hline 37 & 62.5 & $61.7 \pm 0.85$ & $62.5 \pm 0.90$ \\
\hline 42 & 64.4 & $63.6 \pm 0.78$ & $64.0 \pm 0.87$ \\
\hline 47 & 66.1 & $65.9 \pm 0.79$ & $66.6 \pm 0.92$ \\
\hline 52 & 67.9 & $68.0 \pm 0.99$ & $67.7 \pm 0.88$ \\
\hline 57 & 69.4 & $69.6 \pm 0.72$ & $69.4 \pm 0.95$ \\
\hline 63 & 70.5 & $70.9 \pm 0.89$ & $70.5 \pm 0.78$ \\
\hline
\end{tabular}

Analyzing Table 3, we can conclude that the egg weight increases with age of the bird from 47.0-47.5 $\mathrm{g}$ at the beginning to $70.5-70.9 \mathrm{~g}$ by the end of laying.

Feeding the probiotic Levusell SB Plus did not have a significant effect on the egg weight, the difference was in the range of $0.2-1.2 \mathrm{~g}$ or $0.3-2.2 \%$.

Comparison with the norm showed that the greatest deviation from the norm was observed at the beginning of the experiment and amounted to $1.5 \mathrm{~g}$ or $3.1 \%$ in the control group, and $2.0 \mathrm{~g}$ or $4.1 \%$ in the experimental group. Subsequently, the difference in egg weight between the norm and the control group ranged within $0.1-0.8 \mathrm{~g}$ or $0.1-1.3 \%$, in the experimental group $-0.2-0.6 \mathrm{~g}$ or $0.3-1.0 \%$. It should be noted that there was no deviation from the norm in the experimental group at the age of 37, 57 and 63 weeks. Therefore, the use of the probiotic helps to optimize the egg weight of broiler breeders.

One of the key indicators in assessing the productive qualities of hens of the parent flock is the yield of hatching eggs (Table 4).

From the results presented in Table 4 , it follows that there was the lowest yield of hatching eggs in the first month of the experiment $(51.4 \%)$. This is explained by the fact that at the beginning of laying, hens lay small eggs, which are not suitable for incubation, which is confirmed by the data in Table 3 .

Throughout the experiment, except for the first month, it was received more incubation eggs by $0.7-10.6 \%$ from the hens of the experimental group, and the yield of incubation eggs was higher by $0.1-1.6 \%$. In total, during the experiment, the total production of hatching eggs in the control group was 1484760 eggs, which is 53560 eggs or $3.6 \%$ less than in the experimental group. 
The highest yield of hatching eggs in the control group was from 33 to 52 weeks and was $97.2-97.7 \%$, in the experimental group, it was from 33 to 57 weeks - in the range of $97.2-97.9 \%$. On average, over the entire period of the study, the yield of hatching eggs in the experimental group was $0.8 \%$ higher.

Table 4. Total production and yield of hatching eggs.

\begin{tabular}{|c|c|c|c|c|}
\hline \multirow[b]{3}{*}{$\begin{array}{l}\text { Bird age, } \\
\text { weeks }\end{array}$} & \multicolumn{4}{|c|}{ Group } \\
\hline & \multicolumn{2}{|c|}{ control } & \multicolumn{2}{|c|}{ experimental } \\
\hline & $\begin{array}{l}\text { Total yield of } \\
\text { hatching eggs, } \\
\text { pieces. }\end{array}$ & $\begin{array}{l}\text { Yield of } \\
\text { hatching } \\
\text { eggs, \% }\end{array}$ & $\begin{array}{l}\text { Total yield of } \\
\text { hatching eggs, } \\
\text { pieces. }\end{array}$ & $\begin{array}{l}\text { Yield of } \\
\text { hatching } \\
\text { eggs, \% }\end{array}$ \\
\hline $23-27$ & 66390 & 51.4 & 62490 & 51.4 \\
\hline $28-32$ & 242190 & 93.8 & 245400 & 95.3 \\
\hline $33-37$ & 239430 & 97.2 & 242010 & 97.4 \\
\hline $38-42$ & 227010 & 97.7 & 228510 & 97.9 \\
\hline $43-47$ & 207270 & 97.4 & 213990 & 97.8 \\
\hline $48-52$ & 187530 & 97.2 & 199830 & 97.6 \\
\hline $53-57$ & 162750 & 96.3 & 179970 & 97.2 \\
\hline $58-63$ & 152190 & 95.6 & 166120 & 95.7 \\
\hline Total & 1484760 & & 1538320 & \\
\hline On average & & 92.8 & & 93.6 \\
\hline
\end{tabular}

A clear illustration of the yield of hatching eggs per the initial laying hen with a cumulative total is shown in Fig. 2.

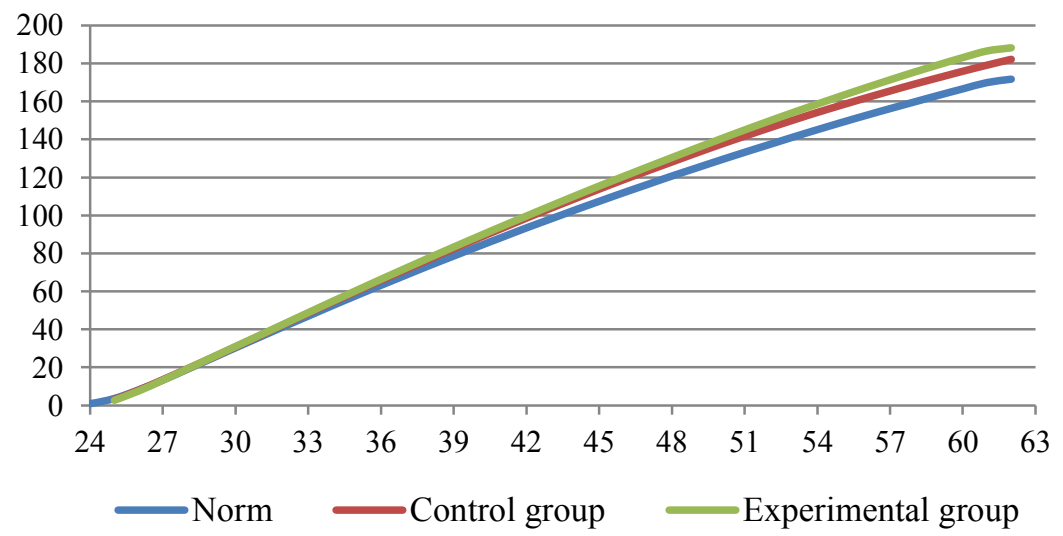

Fig. 2. The yield of hatching eggs per the initial layer with a cumulative total, pcs.

In Fig. 2, it is noticeable that the experimental and control groups exceed the norm in the yield of hatching eggs throughout the experiment. For the entire period of keeping the parent stock from one initial layer of the experimental group, 188.2 hatching eggs were obtained, which is 16.5 pcs. or $9.6 \%$ and 6.0 pcs. or $3.3 \%$ less than the norm and control, respectively.

The use of the probiotic Levusell SB Plus was also reflected in the hatching of chickens (Fig. 3). 


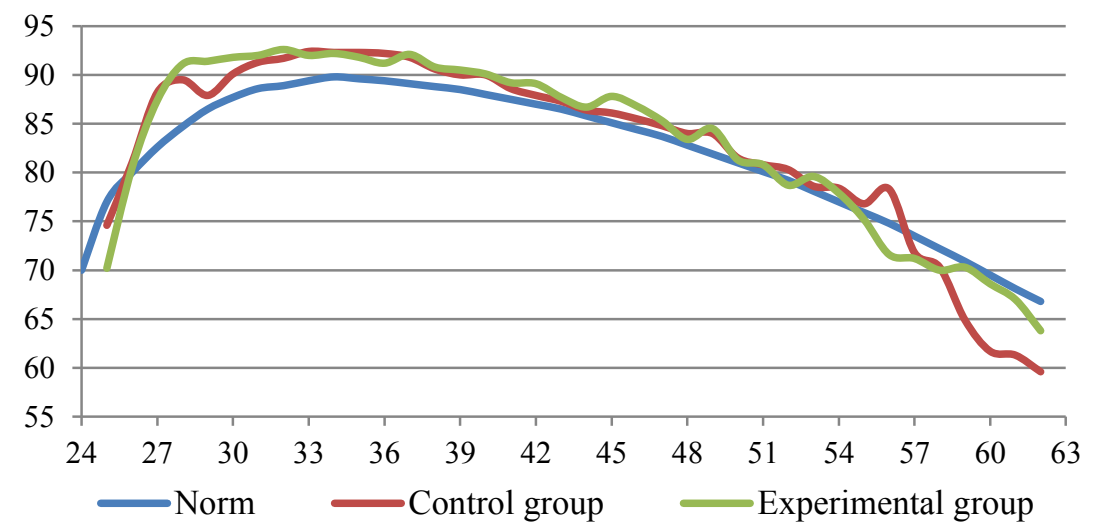

Fig. 3. Hatching of chickens, $\%$.

Based on the data in Fig. 3, we can conclude that the maximum hatching of chickens (more than 80\%) falls on the period from 26 to 52 weeks of age. Almost throughout the entire study, the experimental and control groups exceeded the recommended values, only at the end of the experiment, there was a sharper decrease in hatchability of young birds. So, in the control group, it was observed from week 57, and it was within 1.7-7.8\%, and in the experimental group - from week 55 and only $0.6-3.2 \%$. On average, the hatching of chickens in the control group was $82.0 \%$, which is $1.0 \%$ less than in the experimental group, the chickens of which received the probiotic as part of the diet.

The final indicator, which includes all the main productive qualities of poultry and allows for a more accurate assessment, is the yield of broilers per the initial layer (Table 5).

Table 5. The yield of broilers per the initial layer with a cumulative total, heads.

\begin{tabular}{|c|c|c|}
\hline \multirow{2}{*}{ Bird age, weeks } & \multicolumn{2}{|c|}{ Group } \\
\cline { 2 - 3 } & control & experimental \\
\hline 27 & 10.9 & 10.4 \\
\hline 32 & 36.9 & 37.3 \\
\hline 37 & 63.3 & 64.1 \\
\hline 42 & 87.7 & 88.9 \\
\hline 47 & 109.0 & 111.3 \\
\hline 52 & 127.3 & 130.8 \\
\hline 57 & 141.7 & 146.6 \\
\hline 63 & 150.8 & 156.7 \\
\hline In\% to the control group & & 103.9 \\
\hline Total broiler yield, heads & 1256315 & 1305468 \\
\hline
\end{tabular}

From the data in Table 5, it can be seen that at the beginning of the experiment at the age of 27 weeks of the chickens of the control group, 10.9 chicks were obtained per initial layer, which is 0.5 heads or $4.6 \%$ more compared to the experimental one. In the future, there is a steady increase in the difference in this indicator between the control and experimental groups in favor of the experimental one. Over the period of the experiment, the yield of broilers per the initial layer in the experimental group was 156.7 heads, which exceeds the control group by 5.9 heads or $3.9 \%$.

In general, the broiler yield in the control group was less by 49153 heads or $3.9 \%$ than in the experimental group, the diet of which included the probiotic Levusell SB Plus.

\section{Conclusions}


Thus, the use of the probiotic Levusell SB Plus contributes to an increase in the livability of poultry by $2.1 \%$, an increase in the total production of eggs by $2.7 \%$ and the intensity of egg-laying capacity by $2.4 \%$, optimization of the egg weight, and an increase in the yield of hatching eggs by $0.8-3.3 \%$, hatching of chickens - by $1.0 \%$, and the output of broilers per the initial layer - by $3.9 \%$.

\section{References}

1. I. Egorov, T. Egorova, L. Krivoruchko Probiotics - an alternative to antibiotics in broiler poultry farming, 3, 61-63 (2019)

2. I. Volkova Probiotics as an alternative to feed antibiotics Poultry farming, 2, 10-12 (2014)

3. U. Ramlucken, Santosh O. Ramchuran, G. Moonsamy, R. Lalloo, Mapitsi S. Thantsha, Christine Jansen van Rensburg A novel Bacillus based multi-strain probiotic improves growth performance and intestinal properties of Clostridium perfringens challenged broilers, Poultry Science, 99, 1, 331-341 (2020)https://doi.org/10.3382/ps/pez496

4. J. W. Park, J. S. Jeong, S. I. Lee, I. H. Kim, Effect of dietary supplementation with a probiotic (Enterococcus faecium) on production performance, excreta microflora, ammonia emission, and nutrient utilization in ISA brown laying hens, Poultry Science, 95, 12, 2829-2835, (2016) https://doi.org/10.3382/ps/pew241

5. C.Forte, G.Acuti, E.Manuali, P.Casagrande Proietti, S.Pavone, M.Trabalza-Marinucci, L.Moscati, A.Onofri, C.Lorenzetti, M.P.Franciosini, Effects of two different, probiotics, on microflora, morphology, and morphometry of gut in organic laying hens, Poultry Science, 1, 95, 11, 2528-2535 (2016) https://doi.org/10.3382/ps/pew164

6. Wei-wei Wang, Jing Wang, Hai-jun Zhang, Shu-geng Wu, Guang-hai Qi,Effects of Clostridium butyricum on production performance and intestinal absorption function of laying hens in the late phase of production, Animal Feed Science and Technology, 264, 114476 (2020) https://doi.org/10.1016/j.anifeedsci.2020.114476

7. I.A. Egorov Probiotic in compound feed for broiler chickens Poultry farming, 3. 25-2 (2019)

8. N.H.Eckert, J.T.Lee, D.Hyatt, S.M.Stevens, S.Anderson, P.N.Anderson, R.Beltran, G.Schatzmayr, M.Mohnl, D.J.Caldwell Influence of probiotic administration by feed or water on growth parameters of broilers reared on medicated and nonmedicated diets, Journal of Applied Poultry Research, 1 19, 59-67, (2010) https://doi.org/10.3382/japr.2009-00084

9. Ibiara Correia de Lima Almeida Paz, Ianê Correiade Lima Almeida, Leonardo Thielode La Vega, Elisane LenitaMilbradt, Mariana Rodrigues Borges, Gustavo Henrique CoelhoChaves, Caio Césardos Ouros, Marconi ItaloLourenço da Silva, Fabiana RibeiroCaldara, Raphael LucioAndreatti Filho, Productivity and Well-Being of Broiler Chickens Supplemented With Probiotic, Journal of Applied Poultry Research, 28, 4, 930-942 (2019) https://doi.org/10.3382/japr/pfz054

10. Al-KhalaifaH, Al-NasserA, Al-SurayeeT, Al-KandariS, Al-EnziN, Al-SharrahT, RaghebG, Al-QalafS, MohammedA, Effect of dietary probiotics and prebiotics on the performance of broiler chickens, Poultry Science, 1 98, 10, 4465-4479 (2019) https://doi.org/10.3382/ps/pez282

11. Dariusz Mikulski, Jan Jankowski, Marzena Mikulska, Vanessa Demey, Effects of dietary probiotic (Pediococcus acidilactici) supplementation on productive performance, egg quality, and body composition in laying hens fed diets varying in 
energy density, Poultry Science, 99, 4, 2275-2285

https://doi.org/10.1016/j.psj.2019.11.046

12. NeijatM, ShirleyR B, BartonJ, ThieryP, WelsherA, KiarieE, Effect of dietary supplementation of Bacillus subtilis DSM29784 on hen performance, egg quality indices, and apparent retention of dietary components in laying hens from 19 to 48 weeks of age, Poultry Science, 1 98, Issue 11Pages 5622-5635 (2019) https://doi.org/10.3382/ps/pez324

13. A.A. Ovchinnikov, Yu.V. Matrosova, D.A. Konovalov Productivity of laying hens and the quality of hatching eggs when using probiotics in the diet, 1 (25). P. 105-112 (2019)

14. A.A. Ovchinnikov, L.Yu. Ovchinnikova The use of probiotic feed additives in the diet of rearing stock of table poultry, 9, 13-21 (2019)

15. E.V. Piliukshina, V.N. Khaustov, Economic efficiency of using probiotics in broiler chicken diets ,11 (157), 115-120 (2017)

16. K.Ya. Motovilov, V.N. Khaustov Influence of probiotics on productive qualities and physiological state of broiler chickens Feeding agricultural animals and fodder production, 12, 3-8 (2018)

17. A.S. Kazakov, G.I. Kosse, A.N. Ratoshny Probiotic Levusell SB Plus in the diets of broiler chickens of ISA-15 cross, 63, 139-143 (2016) 